\title{
Impacto Funcional da Lombalgia em Pacientes de um Ambulatório Assistencial de Fisioterapia
}

\author{
Juliane Chaves Santos \\ Mayara Emmanuela da Silva Pereira \\ Selena Dubois Mendes \\ Katia Nunes Sá \\ Coordenadora da Pós-Graduação da Escola Bahiana \\ de Medicina e Saúde Pública (katia- \\ coordpos@bahiana.edu.br) \\ Tipo de Pesquisa \\ Artigo Original \\ Origem da Pesquisa \\ Trabalho de Conclusão de Curso de Fisioterapia da \\ Escola Bahiana de Medicina e Saúde Pública da Bahia \\ Análise do Manuscrito \\ Corpo Editorial da Bahiana \\ Tipo de Análise do Manuscrito \\ Peer Blind Review \\ Recebido em Mai/11 \\ Aprovado em Jun/11
}

\section{Resumo}

Introdução: A lombalgia é uma condição dolorosa que acomete a região entre os últimos arcos costais e as pregas glúteas e é o segundo maior distúrbio álgico que atinge o ser humano. Freqüentemente gera incapacidade funcional, representando grande causa de morbidade, levando muitos trabalhadores ao absenteísmo e gerando elevados custos com sua saúde. Esta dor tem elevado impacto na funcionalidade do portador, tanto que intervenções mais efetivas tem se direcionado mais à recuperação da função do que em atingir a cura. Objetivo: Verificar o impacto funcional da dor lombar em pacientes de um ambulatório assistencial de fisioterapia, em Salvador, Bahia. Métodos: Estudo observacional de corte transversal, com base de dados secundários através da análise de 321 prontuários de pacientes com lombalgia, que utilizou um roteiro estruturado, sendo os resultados analisados através do SPSS versão 14.0 para Windows. Resultados: A dor lombar severa foi a mais frequente $(56,1 \%)$, acometendo predominantemente os adultos $(60,4 \%)$, mulheres $(78,7 \%)$, com elevado impacto funcional $(60,5 \%)$. A incapacidade para realizar atividades de vida diária foi a disfunção mais presente $(59,2 \%)$, sendo que $47,4 \%$ relataram alteração na marcha associada à diminuição da ADM e da FM. Se consideraram independentes $74,1 \%$ da amostra, enquanto que $16,8 \%$ necessitam de auxílio, sendo que $8,4 \%$ dos auxiliados utilizam muletas. Observou-se associação da piora da funcionalidade com a intensidade severa $(\mathrm{p}<0,001)$. Conclusão: Observou-se predomínio álgico severo, em queimação, de forma constante, em adultos do sexo feminino. Estes portadores de lombalgia apresentam importantes alterações nas AVD'S, e na marcha com diminuição da ADM e FM, apesar de serem independentes.

Palavras-Chave: Lombalgia, Dor lombar, Fisioterapia, Funcionalidade

\section{Functional Impact of Low Back Pain Patients in an Ambulatory Care Physiotherapy}

\section{Abstract}

Introduction: Low back pain is a painful condition that affects the region between the last ribs and the gluteal folds and is the second highest painful disorder that affects humans. Often 
leads to disability, representing a major cause of morbidity, leading many workers to generating high absenteeism and health costs. This pain has a high impact on the functionality of the carrier, so that more effective interventions have been directed more to the recovery of function than to achieve a cure. Objective: To assess the functional impact of low back pain patients in an outpatient physical therapy care in Salvador, Bahia. Methods: An observational cross-sectional, based on secondary data through analysis of medical records of 321 patients with low back pain, which used a structured and the results analyzed using SPSS version 14.0 for Windows. Results: The severe back pain was the most frequent $(56.1 \%)$, predominantly affecting adults (60.4\%), women (78.7\%) with high functional impact $(60.5 \%)$. The inability to perform activities of daily living dysfunction was more prevalent (59.2\%), and $47.4 \%$ reported change in gait associated with decreased ROM and FM. $74.1 \%$ considered themselves independent of the sample, while $16.8 \%$ need help, and $8.4 \%$ of the assisted use crutches. An association of functionality with the worsening of severe intensity $(\mathrm{p}<0.001)$. Conclusion: There was a predominance of severe pain, burning, steadily, in adult females. These patients with low back pain have alterations in ADLs, and gait with decreased ROM and FM, although they are independent.

Keywords: Low back pain, low back pain, Physiotherapy, Functionality

\section{Impacto funcional de pacientes con dolor lumbar en una fisioterapia de Atención Ambulatoria}

\section{Resumen}

Introducción: El dolor lumbar es una condición dolorosa que afecta a la región entre las últimas costillas y los pliegues glúteos y es el segundo mayor trastorno doloroso que afecta a los humanos. A menudo conduce a la discapacidad, lo que representa una causa importante de morbilidad, lo que lleva a muchos trabajadores a generar un elevado absentismo y los costes sanitarios. Este dolor tiene un alto impacto en la funcionalidad de la compañía, de modo que las intervenciones más eficaces se han dirigido más a la recuperación de la función de la consecución de una cura. Objetivo: Evaluar el impacto funcional de pacientes con dolor lumbar en la atención ambulatoria la terapia física en Salvador, Bahía. Métodos: Estudio observacional de corte transversal, sobre la base de datos secundaria a través del análisis de los registros médicos de 321 pacientes con dolor de espalda baja, que utiliza un enfoque estructurado y analizado los resultados con el programa SPSS versión 14.0 para Windows. Resultados: El dolor de espalda severo fue la más frecuente (56,1\%), que afectan principalmente a los adultos $(60,4 \%)$, mujeres $(78,7 \%)$ con un alto impacto funcional $(60,5 \%)$. La incapacidad para realizar las actividades de la disfunción vida diaria fue más frecuente $(59,2 \%)$, y el $47,4 \%$ informó el cambio en la marcha asociada con disminución de ROM y FM. $74,1 \%$ se consideran independientes de la muestra, mientras que un $16,8 \%$ necesita ayuda, y el $8,4 \%$ de las muletas asistida. Una asociación de la funcionalidad con el empeoramiento de intensidad severa $(p<0,001)$. Conclusión: Se observó un predominio de dolor intenso, quemazón, constantemente, en mujeres adultas. Estos pacientes con dolor de espalda baja tienen alteraciones en las AVD y la marcha con una disminución de ROM y FM, a pesar de que son independientes.

Palabras clave: Dolor de espalda baja, dolor de espalda, fisioterapia, Funcionalidad 


\section{Impact fonctionnel de patients lombalgiques dans une physiothérapie de soins ambulatoires}

\section{Résumé}

Introduction: La lombalgie est une affection douloureuse qui affecte la région entre les dernières côtes et les plis fessiers et est le deuxième plus trouble douloureux qui affecte les humains. Conduit souvent à l'invalidité, ce qui représente une cause majeure de morbidité, conduisant de nombreux travailleurs à générer un absentéisme élevé et des coûts de santé. Cette douleur a un fort impact sur la fonctionnalité du transporteur, de sorte que des interventions plus efficaces ont été dirigés plus à la récupération de la fonction que de parvenir à une guérison. Objectif: évaluer l'impact fonctionnel de patients lombalgiques dans une thérapie ambulatoire de soins physiques à Salvador de Bahia. Méthodes: Une observation transversale, basée sur des données secondaires à travers l'analyse des dossiers médicaux de 321 patients souffrant de douleurs au bas du dos, qui a utilisé une approche structurée et les résultats analysés à l'aide du logiciel SPSS version 14.0 pour Windows. Résultats: Le mal de dos sévère a été la plus fréquente $(56,1 \%)$, affectant principalement les adultes $(60,4 \%)$, les femmes $(78,7 \%)$ avec un impact fonctionnel élevé $(60,5 \%)$. L'incapacité à effectuer des activités de dysfonctionnement de la vie quotidienne était plus fréquente $(59,2 \%)$, et $47,4 \%$ ont signalé des changements dans la démarche associée à une diminution ROM et FM. 74,1\% se considéraient comme indépendants de l'échantillon, tandis que $16,8 \%$ ont besoin d'aide, et de $8,4 \%$ de l'utilisation des béquilles assistée. Une association de fonctionnalité avec l'aggravation de l'intensité sévère $(\mathrm{p}<0,001)$. Conclusion: Il y avait une prédominance de la douleur sévère, des brûlures, de façon constante, chez les femelles adultes. Ces patients souffrant de lombalgies ont des altérations dans les AVQ, et marche avec une diminution ROM et FM, même si elles sont indépendantes.

Mots-clés: Douleurs lombaires, douleurs lombaires, la physiothérapie, la fonctionnalité

\section{Introdução}

Dor é uma experiência sensorial e emocional desagradável, subjetiva e individual, ${ }^{1}$ referida como um problema de saúde pública, devido ao seu alto potencial de influência psicomotora, incapacidade, absenteísmo e elevados custos. ${ }^{2}$

Atrás apenas da cefaléia, a algia que afeta a região das costas entre os últimos arcos costais e as pregas glúteas, ${ }^{3-5}$ frequentemente causa morbidade e altera a capacidade do indivíduo para desempenhar atividades de vida diária. Associa-se a grande impacto sócioeconômico,5 por acometer a população economicamente ativa, levando ao absenteísmo,6 e causando intenso sofrimento. ${ }^{7}$ Atinge proporções epidêmicas, onde 60 a 80\% da população adulta apresenta ou já apresentou lombalgia incapacitante. ${ }^{8-10}$

A lombalgia causa desconforto e fadiga muscular lombar, durando semanas a meses,10,11 podendo gerar dor local e/ou irradiada para as pernas, diminuir a amplitude de movimento e a força muscular, induzir a vícios posturais, desequilíbrio emocional e problemas financeiros, influenciando na funcionalidade, ${ }^{7}$ designada como a execução de atividades usuais e instrumentais da vida diária sem auxílio, com garantia de uma melhor qualidade de vida.4,12

Cerca de $94 \%$ de lombálgicos crônicos têm comprometimento da atividade profissional.13 E estima-se que mais de 10 milhões de brasileiros sofram com a incapacidade resultante da lombalgia,7 estando a cronicidade, severidade e duração da dor, sexo feminino e idade, associados à mesma, demonstrando a multifatoriedade de fatores envolvidos.11,14

A fisioterapia vem apresentando resultados satisfatórios na recuperação global desses pacientes pela importância do fortalecimento da musculatura com perda de força, do 
alongamento e relaxamento de grupos musculares tensionados e pela reeducação da postura e da precisão do movimento, ${ }^{15}$ pois estes procedimentos modificam a capacidade funcional do indivíduo.

A cronicidade dos sintomas e incapacidade que ocorrem freqüentemente diante da lombalgia têm demonstrado que mais importante do que aliviar a dor, é necessário recuperar a funcionalidade. ${ }^{11}$ Entretanto, a literatura apresenta escassez de dados sobre a funcionalidade, o que restringe a atuação de fisioterapeutas baseada em evidências científicas. Assim, esse estudo objetiva identificar os impactos funcionais da dor lombar em pacientes de um ambulatório docente-assistencial de referência em Salvador, Bahia, a fim de fundamentar as ações interventivas futuras.

\section{Material \& Método}

Estudo observacional de corte transversal, com base de dados secundários, sendo a população estudada pacientes com lombalgia, atendidos em um ambulatório docenteassistencial de fisioterapia, em Salvador, Bahia. A amostra de 321 prontuários estudados foi calculada com base nos parâmetros de erro amostral de 5\%, nível de confiança de $95 \%$, através de um total populacional de 1943 pacientes com o presente diagnóstico confirmado pelo Código Internacional de Doença (CID).

As informações foram coletadas mediante um roteiro estruturado, formulado pelas pesquisadoras, através do contingente de dados de prontuários construídos e preenchidos por integrantes do sistema do ambulatório de fisioterapia. A coleta ocorreu de 08 de abril a 11 de maio de 2011, onde foram selecionados 321 prontuários que haviam sido corretamente preenchidos.

Foras incluídos prontuários de pacientes do ambulatório, atendidos de 2008 a 2011, de ambos os sexos, com idade igual ou superior a 18 anos, que durante as avaliações clínica e funcional, referiram algia lombar. Foram excluídas fichas rasuradas e/ou danificadas, com preenchimento incompleto, portadores de doenças auto-imunes e história de cirurgias ortopédicas envolvendo a coluna lombar.

0 estudo possuiu apenas variáveis categóricas, uma vez que as numéricas também foram categorizadas. As preditoras foram sexo, idade, ocupação, intensidade, local, freqüência, comportamento e característica da dor; e as de desfecho, o diagnóstico funcional e uso de auxiliares locomotores.

A faixa etária foi classificada em jovem, de 18 a 23 anos, adulto de 24 a 59, idoso acima de 60 anos, conforme a Organização Mundial de Saúde. A freqüência da dor em intermitente, constante e esporádica; e o comportamento da dor em um turno, dois turnos, o dia inteiro ou momentânea.

$\mathrm{Na}$ análise descritiva, os dados foram apresentados em freqüências simples e proporções por sexo, faixa etária, entre outros. Utilizou-se como valor de significância estatística um $\mathrm{p} \leq 0,05$. Após a aquisição dos dados, os mesmos foram tabulados, ordenados e descritos estatisticamente através do software Statistical Package for Social Sciences (SPSS), versão 14.0 para Windows.

Algumas variáveis que foram preenchidas na fichas na categoria de "outros", as respostas foram utilizadas, quando possível, a partir de uma recategorização entre as opções da ficha clínica. 0 projeto de pesquisa foi aprovado sob o protocolo n⿳0 $027 / 11$ pelo Comitê de Ética da Escola Bahiana de Medicina e Saúde Pública e respeitou todos os princípios do decreto 196/96 do Conselho Nacional de Saúde. 


\section{Resultados}

A amostra do estudo foi constituída por 321 pacientes, sendo $254(79,1 \%)$ do sexo feminino e adultos $(60,4 \%)$ (Tabela 1). A característica de dor mais freqüente foi em queimação observada em $122(38,0 \%)$ indivíduos. Desta amostra, $152(47,1 \%)$ pacientes apresentaram alteração da marcha associada à diminuição de Amplitude de Movimento (ADM) e da Força Muscular (FM). Destas 152 pessoas, $47(30,9 \%)$ associam os tratamentos fisioterapêutico e medicamentoso, revelando que conforme aumenta a intensidade do sintoma, maior é busca por diferentes associações de tratamentos $(p=0,026)$.

Segundo a funcionalidade, de $298(92,8 \%)$ prontuários preenchidos neste item, 190 $(59,2 \%)$ pacientes apresentam dificuldade nas AVD'S. De $301(93,7 \%)$ prontuários preenchidos sobre nível de dependência, $238(74,1 \%)$ pacientes são independentes e não necessitam de assistência tecnológica (Figuras 1 e 2).

Segundo a manifestação dolorosa, dos 312 (97,1\%) prontuários preenchidos, a intensidade severa foi observada em $180(56,1 \%)$ pacientes e a frequência constante em 153 $(47,7 \%)$. A dor dura o dia inteiro em $68(21,2 \%)$ pacientes, $24(25,3 \%)$ utilizam a associação de fisioterapia com medicamentos, de $44(13,7 \%)$ pacientes com dor em um turno, 13 $(29,5 \%)$ utilizam apenas fisioterapia ou sua associação com medicamentos (Figura 3).

Relacionando a intensidade da dor e suas repercussões, de 180 pacientes com dor severa, $154(85,5 \%)$ são mulheres $(\mathrm{p}<0,001)$, frequência de severidade foi encontrada nos adultos, onde dos 180 pacientes, $33(18,3 \%)$ apresentaram dor em queimação $(p<0,001), 190$ $(59,2 \%)$ pessoas apresentam dificuldade nas AVD'S $(p=0,020)$. Há predomínio da severidade em $109(60,5 \%)$ adultos $(p=0,335)$, percebendo-se que a intensidade relaciona-se com o sexo feminino, característica da dor em queimação e a funcionalidade afetada no desempenho das atividades diárias. Não foram observadas associações com o envelhecimento, uma vez que pacientes idosos apresentam menores queixas e uso de medicamentos. Em relação à severidade, dos $51(28,3 \%)$ pacientes com dor severa e dos $34(29,6 \%)$ com dor moderada $(p=0,036)$, percebeu-se que quanto maior a severidade da dor, maior a utilização de tratamentos (Tabela 2).

Em relação ao comportamento da dor, de 173 (53,8\%) prontuários preenchidos neste item, ambos os sexos têm predominância da dor o dia inteiro em $68(39,3 \% ; p=0,086)$, sendo $53(77,9 \%)$ mulheres e $15(22,1 \%)$ homens, $37(54,4 \%)$ adultos e $27(39,7 \%)$ idosos $(p=0,437)$. A dor momentânea predomina em $39(15,4 \%)$ mulheres, e um turno em $9(13,4 \%)$ homens. Não há relação entre o avançar da idade e a manifestação dolorosa nesta amostra quando se procedeu com a comparação entre as faixas etárias. Relacionando a idade e a característica da dor, de $314(97,8 \%)$ prontuários preenchidos para este item, $52(16,6 \%)$ têm dor em queimação, sendo $27(51,9 \%)$ adultos e $22(42,3 \%)$ idosos $(p=0,851)$.

De 190 (59,2\%) pessoas com dificuldade nas AVD's, duas faixas etárias predominaram $(p=0,454)$ : em adultos $117(61,6 \%)$ e idosos $67(35,3 \%)$, sendo $158(83,2 \%)$ mulheres, e 32 $(16,8 \%)$ homens $(p=0,296)$, sem relação entre o avançar da idade e as alterações funcionais, uma vez que a terceira idade apresenta freqüências menores do que os mais jovens, porém há relação entre o sexo e funcionalidade, revelando que mulheres apresentam maior risco para comprometimento funcional. A dor dura o dia inteiro $(p=0,031)$ em $45(23,7 \%)$ pessoas, apesar de $137(72,1 \%)$ serem independentes $(p=0,039)$, havendo relação entre a funcionalidade, a duração da algia, e a locomoção do indivíduo (Tabela 3).

\section{Discussão}

Ao se verificar os impactos da dor lombar em pacientes que buscam tratamento de fisioterapia, observou-se predomínio álgico severo, em queimação, de forma constante, durante todo o dia, sendo os mais comprometidos, adultos do sexo feminino. Estes portadores 
de lombalgia apresentam importantes alterações nas AVDs, e na marcha com diminuição da ADM e FM, apesar de independentes. No intuito de amenizar a dor e complicações, a maior parte dos lombálgicos, agrega a fisioterapia ao uso de medicamentos.

A intensidade predominante severa discorda dos achados de Ponte, ${ }^{17}$ onde $67 \%$ dos investigados apresentaram dor moderada. Mulheres apresentam altos riscos quanto ao desenvolvimento álgico severo, como verificado no presente estudo, embora superiores aos $54,2 \%$ relatados pela mesma autora. Esta diferença pode ser explicada pela desproporção de mulheres nos dois estudos. As pesquisas têm sugerido que as mulheres apresentam maiores risco e severidade de dor que os homens, devido a sua menor resistência e limiar de dor, características anátomo-funcionais, como menores massa óssea e resistência muscular, articulações mais instáveis, e às grandes cargas ergonômicas, oriundas da dupla jornada de trabalho dentro e fora do ambiente doméstico.3,11,18,19

A lombalgia acomete mais adultos, apesar da não relação com a intensidade, coincidindo com estudo em que $64,9 \%$ pacientes desta faixa etária apresentam maior freqüência de dor lombar. Observa-se que com o avançar da idade ocorre um aumento da resistência à dor, ${ }^{20}$ devido à deterioração fisiológica do sistema nervoso que se inicia por volta dos 30 anos, e ao seu aumento do limiar para dor, além de os idosos apresentarem redução das cargas de trabalho por aposentadoria. Os achados do presente estudo discordam, todavia, de um estudo que encontrou em uma população aleatória entre 18 e 65 anos, maior predomínio de lombalgia no grupo com idade de 50 a $65.3,17$ As diferenças na categorização da idade podem explicar esta discordância.

A dor dos pacientes avaliados apresenta persistência durante todo o dia, concordando com a literatura, ${ }^{17}$ classificada como constante, concordando com Ferreira3 que encontrou em dois grupos submetidos a diferentes tipos de tratamento a predominância do mesmo tipo de dor. Constatou-se que a característica em queimação foi a mais presente, que sugere algia severa e neuropática, não foi condizente com o estudo de Cahú,18 onde apenas 24,59\% de sua amostra apresentou dor irradiada. A divergência poderá ocorrer devido à diferença de caracterização álgica por parte dos dois estudos, uma vez que neste estudo a forma irradiada encontra-se como localização da dor e não como sua característica. Mesmo se ambas as classificações concordassem não haveria homogeneidade de resultados, pois a predominância local da dor foi de forma central, percebendo-se relevância clínica, no momento em que se constata que pessoas com esta localizacão álgica apresentam dificuldade nas AVD'S, não havendo relação entre as variáveis. A associação entre fisioterapia e medicamentos predominou, divergindo da literatura, onde a maioria dos lombálgicos procura tratamento médico e fazem uso apenas de medicações ${ }^{3,11}$. Atualmente, existem evidências que ratificam 0 desempenho fundamental do fisioterapeuta na equipe multidisciplinar21, uma vez que a melhora da funcionalidade do indivíduo tem relação direta com a analgesia, melhora da flexibilidade e força musculares, que consequentemente potencializa a amplitude de movimento. 22,23

Observou-se que pacientes com dor severa, têm maior dificuldade nas AVD'S, corroborando com outro estudo, ${ }^{11}$ onde $27,6 \%$ dos pacientes lombálgicos com 40 anos ou mais já faltou ao trabalho ou deixou de realizar atividades devido à dor. Apesar da dificuldade, $74,1 \%$ dos pacientes se intitulam independentes, podendo, segundo Farinati,12 serem considerados capazes funcionalmente, uma vez que a mesma é a eficácia de se realizar atividades de vida diária sem ajuda. Esses achados não condizem com a literatura que afirma que alterações geradas nos lombálgicos resultam em incapacidade. Ao avaliar a evolução da dor e da capacidade funcional em lombálgicos, tem se observado que grande parte dos pacientes evolui com grande incapacidade funcional, diferença que pode estar associada ao fato desses indivíduos não terem ao seu redor pessoas que os ajudem, não quererem incomodar, ou ainda por associarem ajuda à invalidez. ${ }^{3}$

Encontrou-se predomínio de alteração na marcha associado à diminuição da ADM e FM, concordando com um estudo ${ }^{16}$ que verificou que lombálgicos apresentam diminuição global da força muscular, se comparados com não lombálgicos. Uma das explicações está ligada à 
atrofia muscular resultante normalmente do desuso decorrente da algia, sobrecarregando outras regiões e diminuindo a coordenação e amplitude de movimento em diversas atividades diárias, gerando graves limitações de movimento.16,24 Estudos trazem que a diminuição da flexibilidade muscular principalmente dos isquiotibiais, normalmente gera menor mobilidade no quadril, e sua maior flexibilidade influenciará na amplitude de movimento, potencializando a melhora das atividades de vida diária. ${ }^{24}$

Este estudo revelou que a lombalgia é uma patologia importante e sua intensidade é extremamente relevante para os indivíduos, principalmente por afetar diretamente sua funcionalidade. 0 conhecimento da influência desta na vida funcional destes pacientes possibilita a aquisição de estratégias para tratamentos mais eficazes, de forma direcionada e singular.

A partir destes achados, sugere-se que futuros estudos utilizem a Classificação Internacional de Funcionalidade, Incapacidade e Saúde (CIF), essencial na avaliação e tratamentos fisioterapêuticos por fornecerem um diagnóstico mais amplo do indivíduo e das repercussões sobre as atividades funcionais da lombalgia. ${ }^{25}$

0 estudo apresenta como principal limitação, o não preenchimento total dos prontuários analisados, havendo variação do tamanho amostral de acordo com cada variável analisada, o que pode dificultar a compreensão do leitor, embora todos os ajustes matemáticos necessários tenham sido realizados. 0 modelo de estudo com base em dados secundários impõe dificuldades inerentes ao modelo. Como vantagem, se pode reconhecer que o baixo custo e a facilidade para a coleta dos dados, possibilitaram a definição de fatores importantes para medidas de prevenção, terapêuticas e administrativas que podem contribuir com a redução do sofrimento dos portadores de lombalgia assistidos em um ambulatório cuja razão de ser é a extensão universitária, ou seja, prestação de serviço associada ao ensino e à pesquisa, com vistas a fundamentar melhor a atuação do fisioterapeuta.

\section{Conclusão}

Observou-se predomínio álgico severo, em queimação, de forma constante, em adultos do sexo feminino. Estes portadores de lombalgia apresentam importantes alterações nas AVD'S, e na marcha com diminuição da ADM e FM, apesar de serem independentes. Conclui-se, portanto, que existe alta interferência da dor lombar, quando severa, na funcionalidade do indivíduo, gerando alterações nas atividades de vida diária, embora estas limitações não gerem, na amostra estudada, a necessidade de auxílio na execução de tarefas cotidianas. Também foi observada associação destas alterações com o sexo feminino e a manifestação sensitiva em queimação. Sugere-se um aprofundamento na integração da assistência com a pesquisa, para um aprimoramento mais qualificado da reabilitação funcional e prática clínica em fisioterapia baseada em evidências.

\section{Referências}

1. OKIFUJI, Akiko; TURK, Dennis. "Psychological Factors in Chronic Pain: Evolution and Revolution" In: FERREIRA, Maria S M. Variáveis Psicológicas na Lombalgia Crônica: um estudo com doentes em Tratamento de Fisioterapia e Acupuntura, (Dissertação de Doutorado em Psicologia Área de Especialização em Psicologia da Saúde) - Instituto de Educação e Psicologia, Portugal: Universidade do Minho, 2009, p. 28. (345 p)

2. KARINE, Muniz P; \& SILVANA, Nair L. "Quem Sente é a Gente, mas é Preciso Relevar: a lombalgia na vida das trabalhadoras do setor têxtil de Blumenau- Santa Catarina", São Paulo-SP: Saúde e Sociedade, 19(2): 405-17. 2010.

3. FERREIRA, Maria S M. Variáveis Psicológicas na Lombalgia Crônica: Um estudo com doentes em Tratamento de Fisioterapia e Acupuntura, (Dissertação de Doutorado em Psicologia Área de 
Especialização em Psicologia da Saúde) - Instituto de Educação e Psicologia, Portugal: Universidade do Minho, 2009, $345 \mathrm{p}$.

4. CECIN, Hamid A. "Proposição de uma reserva anatomofuncional, no canal raquidiano, como fator interferente na fisiopatologia das lombalgias e lombociatalgias mecânico-degenerativas", Revista da Associação Médica Brasileira, 4(43): 295-310. 1997.

5. HILDEBRANDT, Vincent A. "Back pain in the work population: prevalence rates in Dutch trades and professions", Ergonomics, 38(6): 1283-98. 1995.

6. FREIRE, Marlene. "O efeito do condicionamento físico pela caminhada, na dor Lombar Crônica", In: FERREIRA, Maria S M. Variáveis Psicológicas na Lombalgia Crônica: um estudo com doentes em Tratamento de Fisioterapia e Acupuntura. (Dissertação de Doutorado em Psicologia Área de Especialização em Psicologia da Saúde) - Instituto de Educação e Psicologia, Portugal: Universidade do Minho, 2009. p. 63. (345 p)

7. GoldenfuM, Marco A; HELFENSTEIN-JR, Milton; \& SIENA, César. "Lombalgia Ocupacional", Revista da Associação Médica Brasileira, 5(56): 583-89. 2010.

8. DEYO, Richard; \& WEINSTEIN, James. "Low back pain”, New England Journal Medicine, 344(5): 36370. 2001

9. BIERING-SORENSEN, Fin. "A prospective study of low back pain in a general population", Scandinavian Journal of Rehabilitation Medicine, 15(2): 71-79.1983.

10. ANDERSSON, Gunnar. "Epidemiological features of chronic low-back pain", The Lancet, 354(9178): 581-85. 1999.

11. FASSA, Ana C G; SILVA, Marcelo C; VALLE, Neiva C J. "Dor lombar crônica em uma população adulta do Sul do Brasil: prevalência e fatores associados", Caderno de Saúde Pública, 20(2): 377-85. 2004.

12. FARINATI, Paulo. “Avaliação da autonomia do idoso: definição de critérios para uma abordagem positiva a partir de um modelo de interação saúde-autonomia. Arquivos de Geriatria e Gerontologia", Vol. 1, pp. 1-9, 1997. In: ALVES, Luciana et al. "A influência das doenças crônicas na capacidade funcional dos idosos do Município de São Paulo, Brasil”, Rio de Janeiro-RJ: Caderno de Saúde Pública, 23(8): 1924-30. 2007.

13. TEIXEIRA, Manoel Jacobsen et al. Dor no Brasil: Estado atual e perspectivas. In: CRUZ, Diná A L; KRELING, Maria C G D; PIMENTA, Cibele A M. "Prevalência de dor crônica em adultos", Revista Brasileira de Enfermagem, 59(4): 509-13. 2006.

14.POLATIN, Peter; et al. "A prospective study of waddell signs in patients with chronic low back pain: When they may not be predictive", Spine, 22(14): 1618-22.1997.

15. DINA, Thomas; et al. "Abnormal magnetic-resonance scans of the lumbar spine in asymptomatic subjects", In: CECIN, Hamid A. "Proposição de uma reserva anatomofuncional, no canal raquidiano, como fator interferente na fisiopatologia das lombalgias e lombociatalgias mecânico-degenerativas", Revista da Associação Médica Brasileira, 4(43): 295-310. 1997.

16. MATHEWS, John A. “Bone and Joint Diseases. Backache”, British Medical Journal, 1: 432-34. 1997.

17. PONTE, Carla. "Lombalgia em cuidados de saúde primários. Sua relação com características sociodemográficas", Revista Portuguesa de Clínica Geral, 21: 259-67. 2005.

18. CAHÚ, F M G; SIQUEIRA, Gisela R; VIEIRA, Ricardo A G. "Ocorrência de lombalgia em Fisioterapeutas da cidade de Recife, Pernambuco", Revista Brasileira de Fisioterapia, 12(3): 222-27. 2008.

19. FILLINGIM, Roger. "Sex-related influences on pain, a Review of Mechanisms and clinical implications", In: FERREIRA, Maria S M. Variáveis Psicológicas na Lombalgia Crônica: Um estudo com doentes em Tratamento de Fisioterapia e Acupuntura. (Dissertação Doutorado em Psicologia Área de Especialização em Psicologia da Saúde) - Instituto de Educação e Psicologia, Portugal: Universidade do Minho, 2009.

20. DIAS-DA-COSTA, Juvenal S; et al. "Dor lombar em usuários de um plano de saúde: prevalência e fatores associados", Rio de Janeiro-RJ: Caderno de Saúde Pública, 24(9): 2115-22. 2011. 
21. DALBERTO, Vânia; OLIVEIRA, Michele; \& MACEDO, Christiane S G. "Prevalência de lombalgia em costureiras e correlação com a qualidade de vida e incapacidade", Revista Arquivos de Ciências da Saúde, 8(2): 111-20.2004.

22. CRUZ, Eduardo B; \& SERPA, Rui. "Padrões de dor lombar: categorização dos sinais, sintomas e restrições da capacidade em utentes com dor lombar", In: FERREIRA, Maria S M. Variáveis Psicológicas na Lombalgia Crônica: Um estudo com doentes em Tratamento de Fisioterapia e Acupuntura. (Dissertação Doutorado em Psicologia Área de Especialização em Psicologia da Saúde)Instituto de Edu cação e Psicologia, Portugal: Universidade do Minho, 2009, 345 p.

23. ANDRADE, Fernanda M; DEBIAGI, Polyana C; \& MACEDO, Christiane S G. "Efeito do isostretching na resistência muscular de abdominais, glúteo máximo e extensores de tronco, incapacidade e dor em pacientes com lombalgia", Revista Fisioterapia em Movimento, 23(1): 113-20. 2010.

24. BIGOLIN, Simone E; \& MACHADO, Guilherme F. "Estudo comparativo de casos entre a mobilização neural e um programa de alongamento muscular em lombálgicos crônicos", Revista Fisioterapia em Movimento, 23(4): 545-54.2010.

25. Organização Mundial de Saúde. Classificação Internacional de Funcionalidade, Incapacidade e Saúde-CIF. Direção Geral de Saúde, pp. 7-29. 2004. (Disponível em: <www.inr.pt/uploads/ docs/cif/CIF_port_\%202004.pdf>. Acesso em 22/07/2011).

\section{Apêndice}

Tabela 1

Distribuição clínica de 321 pacientes de Fisioterapia, em Salvador-BA, entre 2008 e 2011 ,.

\begin{tabular}{c|c|c}
\hline Variáveis & $\mathbf{N}$ & \% \\
\hline Gênero & & 20,9 \\
\hline Masculino & 67 & 79,1 \\
\hline Feminino & 254 & 0,9 \\
\hline Jovem & 3 & 60,4 \\
\hline Adulto & 194 & 36,5 \\
\hline Idoso & 117 & 2,2 \\
\hline Não Informado & 7 & \\
\hline Tratamentosia Atuais & & 28,3 \\
\hline Fisioterapia e Medicamentos & 91 & 24,0 \\
\hline Medicamento & 77 & 23,1 \\
\hline Fisioterapia & 74 & 13,1 \\
\hline Outros Tratamentos & 42 & 11,5 \\
\hline Não Informado & 37 & \\
\hline Diagnóstico Funcional Alteração & & 47,4 \\
\hline Marcha, ADM e FM* & 152 & 9,4 \\
\hline Outras regiões** & 30 & 7,1 \\
\hline Outras Funções*** & 23 & 0,3 \\
\hline Sem Alteração & 115 & 35,8 \\
\hline Não Informado & & \\
\hline
\end{tabular}


SANTOS, J C et al. "Impacto Funcional da Lombalgia ..."

\begin{tabular}{c|c|c}
\hline Local da Dor & & 38,0 \\
\hline Central & 122 & 32,1 \\
\hline Bilateral & 103 & 7,5 \\
\hline Bilateral+Irradiada & 24 & 6,5 \\
\hline Irradiada & 21 & 5,3 \\
\hline Lateral & 17 & 6,8 \\
\hline Outras regiões & 22 & 3,8 \\
\hline Não Informado & 12 & \\
\hline
\end{tabular}




\begin{tabular}{c|c|c}
\hline Característica da Dor & & 16,2 \\
\hline Queimação & 52 & 9,7 \\
\hline Cansada & 31 & 7,2 \\
\hline Peso & 23 & 6,9 \\
\hline Q+C+P**** & 22 & 6,2 \\
\hline Latejante & 20 & 48,5 \\
\hline Outras classificações & 156 & 5,3 \\
\hline Não Informado & 17 & \\
\hline
\end{tabular}

$\mathrm{N}$ (número de pacientes); *ADM (Amplitude de Movimento);

FM (Força Muscular); ** Cervical, MMSS (Membros Superiores); ***Pentear o cabelo; **** Q (Queimação), C (Cansada), P (Peso).

Figura 1

Nível de dependência de 321 pacientes de Fisioterapia, em Salvador-BA entre 2008 e 2011

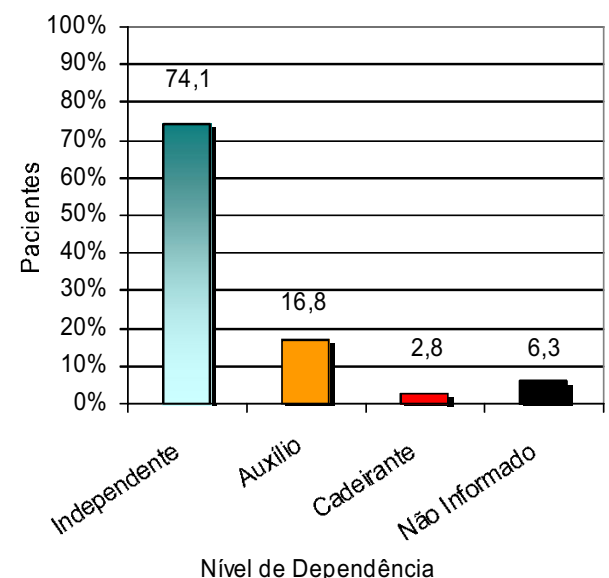

Figura 2

Assistência Tecnológica de 321 pacientes de Fisioterapia, em Salvador-BA, entre 2008 e 2011

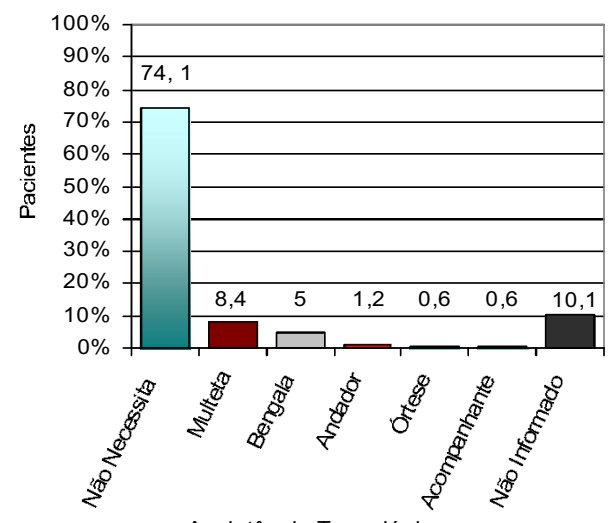

Assistência Tecnológica 
Tabela 2

Associação da intensidade da dor e características observadas, de 321 pacientes atendidos no ambulatório de Fisioterapia, em Salvador-BA, entre 2008 e 2011

\begin{tabular}{|c|c|c|c|c|}
\hline \multirow[b]{2}{*}{ Variáveis } & \multicolumn{3}{|c|}{ Intensidade da Dor - Perdidos $=11(3,4 \%)$} & \multirow[t]{2}{*}{$p \leq 0,05$} \\
\hline & $\begin{array}{c}\text { Severa } \\
\mathrm{n}=180(56,1 \%)\end{array}$ & $\begin{array}{c}\text { Moderada } \\
\mathrm{n}=115(35,8 \%)\end{array}$ & $\begin{array}{c}\text { Leve } \\
\mathrm{n}=15(4,7 \%) \\
\end{array}$ & \\
\hline Sexo & & & & 0,001 \\
\hline Feminino & $154(85,6 \%)$ & $82(71,3 \%)$ & $8(53,3 \%)$ & \\
\hline Masculino & $26(14,4 \%)$ & $33(28,7 \%)$ & $7(46,7 \%)$ & \\
\hline Faixa Etária & & & & 0,335 \\
\hline Jovem & $1(3,4 \%)$ & $1(2,7 \%)$ & $1(6,7 \%)$ & \\
\hline Adulto & $109(60,5 \%)$ & $67(58,2 \%)$ & $12(80,0 \%)$ & \\
\hline Idoso & $65(36,1 \%)$ & $45(39,1 \%)$ & $2(13,3 \%)$ & \\
\hline História Funcional & & & & 0,020 \\
\hline Dificuldade AVD'S* & $109(60,5 \%)$ & $66(57,4 \%)$ & $5(33,3 \%)$ & \\
\hline Não apresenta alteração & $37(20,6 \%)$ & $34(29,6 \%)$ & $7(46,7 \%)$ & \\
\hline Limitação & $19(10,6 \%)$ & $8(7,0 \%)$ & $2(13,3 \%)$ & \\
\hline Perdidos & $15(8,3 \%)$ & $7(6,0 \%)$ & $1(6,7 \%)$ & \\
\hline Aparelho Locomotor & & & & 0,744 \\
\hline Independente & $132(73,3 \%)$ & $88(76,5 \%)$ & $9(60,0 \%)$ & \\
\hline Auxílio & $28(15,6 \%)$ & $19(16,5 \%)$ & $5(33,3 \%)$ & \\
\hline Cadeirante & $6(3,3 \%)$ & $3(2,6 \%)$ & - & \\
\hline Perdidos & $14(7,8 \%)$ & $5(4,4 \%)$ & $1(6,7 \%)$ & \\
\hline Auxílio Locomotor & & & & 0,650 \\
\hline Não Necessita & $132(73,3 \%)$ & $88(76,5 \%)$ & $4(26,6 \%)$ & \\
\hline Muleta & $15(8,3 \%)$ & $8(7,0 \%)$ & - & \\
\hline Bengala & $7(3,9 \%)$ & $6(5,2 \%)$ & $9(60,0 \%)$ & \\
\hline Órtese & $1(0,6 \%)$ & - & $1(6,7 \%)$ & \\
\hline Acompanhante & $1(0,6 \%)$ & - & - & \\
\hline Perdidos & $24(13,3 \%)$ & $13(11,3 \%)$ & $1(6,7 \%)$ & \\
\hline Característica da Dor & & & & $<0,001$ \\
\hline Queimação & $33(18,3 \%)$ & $18(15,7 \%)$ & $1(6,7 \%)$ & \\
\hline Cansada & $12(6,7 \%)$ & $17(14,8 \%)$ & $2(13,3 \%)$ & \\
\hline Peso & $12(6,7 \%)$ & $6(5,2 \%)$ & $3(20,0 \%)$ & \\
\hline $\mathrm{Q}+\mathrm{C}+\mathrm{P}^{* * * *}$ & $16(8,9 \%)$ & $5(4,3 \%)$ & - & \\
\hline Latejante & $14(7,8 \%)$ & $6(5,2 \%)$ & - & \\
\hline Outras & $88(48,8 \%)$ & $58(50,5 \%)$ & $7(46,7 \%)$ & \\
\hline Perdidos & $5(2,8 \%)$ & $5(4,3 \%)$ & $2(13,3 \%)$ & \\
\hline
\end{tabular}

